AIDS. 2018 September 24; 32(15): 2103-2111. doi:10.1097/QAD.0000000000001951.

\title{
Modelling interaction between HIV-1 Nef and calnexin
}

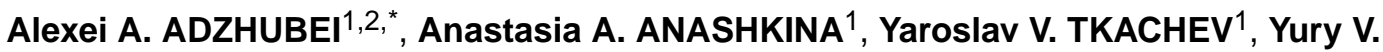 \\ KRAVATSKY ${ }^{1}$, Tatiana PUSHKARSKY ${ }^{2}$, Amol KULKARNI ${ }^{3}$, Alexander A. MAKAROV ${ }^{1}$, and \\ Michael I. BUKRINSKY 2 \\ ${ }^{1}$ Engelhardt Institute of Molecular Biology, Russian Academy of Sciences, 119991 Moscow, \\ Russia \\ ${ }^{2}$ George Washington University School of Medicine and Health Sciences, Washington, DC 20037, \\ USA \\ ${ }^{3}$ Howard University College of Pharmacy, Washington, DC 20059, USA
}

\section{Abstract}

Background: HIV-associated atherosclerosis is a major co-morbidity due in part to systemic effects of the virus on cholesterol metabolism. HIV protein Nef plays important role in this pathology by impairing maturation of the main cellular cholesterol transporter, ABCA1. ABCA1 maturation critically depends on calnexin, an integral endoplasmic reticulum membrane chaperone, and Nef binds to the cytoplasmic domain of calnexin and impairs interaction of calnexin with ABCA1. Overarching goal of the present study was to model Nef-calnexin interaction interface, and identify small molecule compounds potentially inhibiting this interaction.

Methods: Molecular dynamics was utilised to build structure model of calnexin cytoplasmic domain, followed by global docking combined with application of QASDOM software developed by us for efficient analysis of receptor-ligand complexes. Structure-based virtual screening was performed for all sites identified by docking. A soluble analogue of a compound from the screening results list was tested for ability to downregulate ABCA1.

Results: We identified major interaction sites in calnexin and reciprocal sites in Nef. Virtual screening yielded a number of small-molecule compounds potentially blocking a calnexin site. Interestingly, one of the compounds, NSC13987, was previously identified by us as an inhibitor targeting a Nef site. An analogue of NSC13987, AMS-55, potently reversed the negative effect of Nef on ABCA1 abundance.

\footnotetext{
*To whom correspondence should be addressed: Dr. Alexei A. Adzhubei, Engelhardt Institute of Molecular Biology RAS, Vavilov St 32, Moscow 119991, Russia; alexei.adzhubei@eimb.ru.

Alexei A. Adzhubei: alexei.adzhubei@eimb.ru

Anastasia A. Anashkina: anastasya.anashkina@gmail.com

Yaroslav V. Tkachev: yat.imb@gmail.com

Yury V. Kravatsky: jiri@eimb.ru

Tatiana Pushkarsky: tpushk@gwu.edu

Amol Kulkarni: amol.kulkarni@Howard.edu

Alexander A. Makarov: aamakarov@eimb.ru

Michael I. Bukrinsky: mbukrins@gwu.edu
} 
Conclusions: We have modelled Nef-calnexin interaction, predicted small molecule compounds that can potentially inhibit this interaction, and experimentally tested one of these compounds, confirming its effectiveness. These findings provide a platform for searching for new therapeutic agents to treat HIV-associated co-morbidities.

\section{Keywords}

Nef; calnexin; interaction; HIV-1; structure modelling; molecular dynamics; docking; virtual screening; QASDOM server

\section{Introduction}

HIV-associated cardio-vascular disease is a major co-morbidity which affects a large number of HIV-infected subjects, both untreated and treated with anti-retroviral therapy [1]. There are multiple mechanisms responsible for this co-morbidity, but one that can be directly linked to HIV infection is the systemic effects of the virus on cholesterol metabolism. Our studies demonstrated that the HIV protein Nef impairs cholesterol efflux mediated by the cholesterol transporter ATP-Binding Cassette A1 (ABCA1), leading to lipid accumulation in macrophages and their conversion into the 'foam' cells [2] [3] . Importantly, this activity of Nef targeted not only HIV-infected cells, but also uninfected, bystander cells of multiple tissues, which were affected by Nef released into the blood stream [4]. Studies in animal models demonstrated that this activity of Nef may be responsible for hypoalphalipoproteinemia and high risk of atherosclerosis observed in HIV-infected subjects [4] [5] [6]. Of note, Nef can be detected in plasma of HIV-infected patients, both untreated and treated with cART [7] [8] [9], providing a possible explanation for persistence of HIVassociated cardio-vascular disease in HIV-infected subjects with undetectable viral load.

Our recent study identified calnexin, an integral endoplasmic reticulum (ER) membrane lectin-like chaperone, as a key player in the mechanism of Nef-mediated inhibition of ABCA1 and cholesterol efflux [10]. Calnexin is a type I transmembrane protein composed of three parts: a lumenal fragment consisting of a globular domain responsible for the interaction with carbohydrates and a proline-rich tandem sequence repeat domain (the P domain) involved in protein-protein interactions, a transmembrane domain, and a cytoplasmic domain [11] [12]. Biochemical analysis demonstrated that Nef binds to the cytoplasmic domain of calnexin and impairs interaction of calnexin with ABCA1 (supposedly by inducing a conformational change in calnexin), thus affecting ABCA1 maturation [13]. Molecular modeling predicted the interaction site in Nef structure and biochemical analysis confirmed that lysine residues in positions 4 and 7 in Nef were essential for interaction with calnexin [13].

\section{Methods}

\section{Calnexin Cytoplasmic Domain and Nef Model Structures}

Models of Nef and calnexin cytoplasmic domain were built by us previously as described in [13]. Nef modelling was performed for the target sequence P03407 (HIV-1 group M subtype B isolate ARV2/BRU), calnexin modelling for the P27824 (CALX_HUMAN) human 
calnexin. The available model of calnexin cytoplasmic domain was used as a starting structure for molecular dynamics (MD) calculations.

\section{Molecular Dynamics}

Energy minimization, equilibration and production runs were performed with NAMD software [14]. Initial structure preparation and trajectory post-processing were done with VMD software package [15]. Applied force field parameter set for protein was CHARMM27 with CMAP dihedral correction [16] [17]. Single protein molecule was placed within the rectangular unit cell of size $78 \times 76 \times 70 \AA$, and solvated using TIP3P molecules [18] [19]. System net charge was neutralized by adding $\mathrm{Na}^{+}$and $\mathrm{Cl}^{-}$ions. $\mathrm{NaCl}$ concentration was $0.15 \mathrm{M}$ to mimic physiological ionic strength. Simulation was run with periodical boundary conditions, and particle mesh Ewald (PME) summation method was used to compute long-range electrostatics. Integration time step was $1 \mathrm{fs}$. System was initially minimized for 5000 steps, and gradually heated to 300K in 64000 steps. Then, pressure control was switched on, system was equilibrated for $300 \mathrm{ps}$ at pressure target of 1.01325 bar, and production run was initiated to obtain trajectory of $104 \mathrm{~ns}$ total length. For production run, pressure and temperature in course of simulation were held constant using a combination of the Nose-Hoover constant pressure method [20] [21] and Langevin dynamics with collision frequency of $5.0 \mathrm{ps}^{-1}$. System coordinates were saved every $2 \mathrm{ps}$. Computations were performed on 32-core Intel(R) Xeon(R) CPU E5-2670 server. Plots were produced using RStudio software, and Matplotlib Python package [22] [23].

Prior to trajectory analysis, implicit water was stripped from trajectory, and trajectory was downsampled to $10 \mathrm{ps}$ per frame, to reduce size. Initial $32 \mathrm{~ns}$ of the trajectory was omitted to avoid non-equilibrium transient effects (Fig. S1). Principal component analysis and clustering of protein conformations obtained from MD trajectory was carried out using ' $R$ ' statistical software [24] with Bio3d library [25].

\section{Docking}

Global docking was performed using servers Cluspro, HEX, SwarmDock, and Zdock, with calnexin and Nef structures submitted to all servers as receptor and ligand accordingly. The final dataset of 40 models included 10 best Nef-calnexin docking models from each server. The dataset was then submitted to the locally installed server QASDOM [26] (http:// qasdom.eimb.ru) for analysis of interactions, identification of binding sites, and model ranking. A new functionality has been developed to compute maps of receptor-ligand interactions density, implemented in the in-house version of QASDOM and used in the analysis. To select a biologically meaningful 'good' subset from the full dataset of docking models, one must take into account that calnexin cytoplasmic domain connects to the transmembrane domain and Nef interacts with the ER membrane. The following criteria have been applied to reflect this. In the complex the positions of calnexin and Nef (1) should be such that their N-termini point in the same direction, and (2) they are located so as to make possible connection to the membrane. Then the 'good' subset was submitted to QASDOM in order to perform model ranking, and obtain contact maps and interaction sites. Interaction sites in calnexin and Nef were selected according to the QASDOM criteria as linear clusters (stretches of sequence) with the number of residue interactions greater than 
the dataset median, and where the number of atomic interactions was greater than the median for the dataset.

\section{Virtual Screening}

Docking-based (structure-based) virtual screening was performed with locally installed software package AutoDock VINA 1.1.2 (http://vina.scripps.edu/) [27]. Structures of the target sites for virtual screening were prepared by the adt program (AutoDockTools). Computations were performed on a 32-core Intel(R) Xeon(R) CPU E5-2670 LINUX server. As a database of low molecular weight compounds, the publicly accessible ZINC database (http://zinc.docking.org/) [28] was used. For compatibility with our our previous work [13] we used the NCI Plated 2007 dataset of 139,735 compounds (http://zinc.docking.org/ catalogs/ncip).

\section{Development and Experimental Testing of AMS-55}

In spite of its potent NEF-inhibitory activity, NSC13987 (Fig. S2A) is not an attractive target for drug development because of its extreme lipophilicity and poor aqueous solubility. Molecular modeling studies [13] revealed that both anthaquinone and the secondary amine in NSC13987 are essential for the biological activity. Therefore, our initial efforts were focused on chemical alterations to the benzanthrone moiety as a way to augment the aqueous solubility. AMS-55 represents the first, proof-of-concept compound featuring the replacement of benzanthrone moiety with less lipophilic naphthalene ring. It also contains a polar secondary amide group to further improve the aqueous solubility. Guided by the molecular modeling studies, our research was focused on the introduction of the polar carboxylic acid group to the core of AMS-55 (Fig. S2A). Chemical synthesis of AMS-55 is described in Fig. S2B. Aryl bromide 1 was converted into the corresponding carboxylic acid via Pd-catalyzed carbonylation. The latter was coupled with aniline using a peptide coupling agent, EDCI. Deprotection of the Boc-group, followed by the reaction of the resultant primary amine with commercially available bromo-anthroquinone furnished the desired compound, AMS-55. AMS-55 was tested for the ability to block the Nef-mediated downregulation of ABCA1, which was previously shown by us to depend on the blockade by Nef of the interaction between calnexin and ABCA1 [10]. HeLa-ABCA1 cells [29] were transfected with Nef-expressing or empty vector, treated with AMS-55 $(1 \mu \mathrm{M})$, and tested for plasma membrane-associated ABCA1 by Western blot.

\section{Results}

Experimentally solved structure of calnexin is available only for the lumenal domain, and in order to obtain three-dimensional structure of calnexin cytoplasmic domain we performed its modelling [13] with several servers implementing different methods. The models were ranked according to their quality, and the best model structure was used in subsequent round of expert modelling, producing the final model structure. The resulting model had a loosely folded globular structure, Fig. S3. This structure has been used in the current study as a starting point for building a new high quality model utilising molecular dynamics (MD) simulation. 
The most stable model of the protein structure was extracted from the resulting MD trajectory (Fig. S1). Trajectory was subjected to clustering analysis to split it into few clusters of the structurally similar conformations. Relative stability of the conformations was estimated by the amount of MD time the protein spent within the same cluster (residence time), and the cluster density (i.e., RMSD). Therefore, the most stable conformation is the one from which the structure does not deviate significantly for a longest time period during MD run. Protein backbone conformation space, defined by the set of Ramachandran angles $\left(\varphi_{i}, \psi_{i}\right)$ for all 90 backbone residues, has a dimensionality of 178 . To make the problem addressable, the dimensionality was reduced to 4 principal components comprising largest variation. Agglomerative hierarchical clustering using Ward's method was then applied to classify the conformations into 10 groups of structurally similar ones. Conformation possessing minimum root mean square (RMS) difference against the average was selected from each group as a representative. Figure 1 summarizes the clustering analysis results. Three largest clusters of conformations are marked G, F, and C. Cluster G was found to be the densest (with the least variance), and to have the longest residence time of $24 \mathrm{~ns}$ (33\% of total analyzed trajectory length, vs. $18 \%$ for F, and $11 \% \mathrm{C}$ ), and corresponding representative conformation has been selected as the final model (shown in Fig. S3). In comparison with the initial structure there were no dramatic changes. $\mathrm{N}$ - and $\mathrm{C}$-terminal parts of the structure have folded over onto the alpha-helical core resulting in a more tightly packed structure. The structure is stabilised by a network of inter-chain hydrogen bonds, including three bundles that are formed by multiple hydrogen bonds interconnecting residues located in distant segments of the structure (Fig. S4). Overall the MD results showed that the initial calnexin cytoplasmic domain model was sufficiently robust and though it underwent structure rearrangement, that did not alter the basic fold of the protein.

Nef - calnexin interaction modelling has been performed by global docking using the approach which is based on concurrent predictions with different docking servers. The combined data then includes models built with unrelated algorithms and therefore, overall, it is more reliable than if a single server is used. To process such data and identify representative models that incorporate the most frequently occurring residue contacts in the dataset, we developed the QASDOM meta-server [26]. Results of the interactions data analysis include identification of the representative interaction sites and interacting residues in the receptor and ligand, shown in the map of Nef - calnexin contacts and diagrams of the linear contact clusters (Fig. 2 and Interactions Map Supplemental file). We have then formed a subset of biologically relevant 'good' docking models as described in the Methods section. Relative positions of clusters of residue interactions for the full and 'good' datasets are in agreement, showing that the 'good' dataset adequately represents the full data. We have identified three major interaction sites in Nef (Fig. 3), which is in agreement with our previous work [13]. The results confirm high frequency of contacts for the Nef N-terminal interaction site I including residues TRP5-ARG8 that were used for our previous run of virtual screening [13]. An extended site II was identified as a major site interacting with calnexin. Part of this site is formed by a left-handed polyproline-II helix (PPII) GLY71GLN77, which is known as a preferable structure for protein-protein interactions [30] [31]. A range of interaction sites are present in calnexin in different models. Of these, consecutive sites A and B interact with all three sites in Nef (Fig. 3). Overall these sites display the 
highest number of atomic interactions, including high number of interactions with the Nef $\mathrm{N}$-terminal site $\mathbf{I}$. On the balance of number of contacts, calnexin sites $\mathbf{A}, \mathbf{B}$ and $\mathbf{E}$, as well as Nef site II represent promising targets for docking-based virtual screening.

Virtual screening of compounds targeting Nef and calnexin sites has been performed using locally installed docking program Vina. For compatibility, as in our previous work where we performed virtual screening for Nef site I [13], we have used compounds from the Zinc NCI Plated 2007 dataset. Screening has been set up for the segments covering interaction sites AB, B, E in calnexin, and sites II and II-III in Nef (Fig. 4A). Ten putative ligands were identified for each segment and prioritized according to the Vina ranking; Vina docking of the top-ranking compounds to calnexin structure from the Nef-calnexin complex is shown in Fig. 4B. Full list of the identified small-molecule drug candidates is presented in Tables S1 and S2. Excitingly, one of these compounds, ZINC03953858 (NSC13987), is the compound that was previously predicted by us and confirmed experimentally as inhibitor of the Nef site I [13].

The NSC13987 compound, while effective in inhibiting Nef-calnexin interaction [13], has poor solubility, hampering its further development for therapeutic applications. Therefore, in this study we have developed an analog of NSC13987, AMS-55 (Fig. 5A and Fig. S2). Docking to major calnexin and Nef interaction sites showed that though the location of AMS-55 at the sites was similar to that of NSC13987, relative positioning of some of the chemical groups was different (Fig. S6). AMS-55 was tested for the ability to reverse the Nef-mediated decrease of plasma membrane ABCA1. Results in Fig. 5B show that relative abundance of plasma membrane ABCA1 in HeLa-ABCA1 cells transfected with Nef and treated with AMS-55 was reversed to levels in untransfected cells. No cytotoxicity of AMS-55 was observed in the tested range $(0.1-10 \mu \mathrm{M})$. Given that decrease of plasma membrane ABCA1 is responsible for all Nef-mediated effects on cholesterol metabolism (inhibition of cholesterol efflux, increase of abundance of lipid rafts, decrease of HDL levels), this result suggests that AMS-55 can be considered a candidate for development as a therapeutic agent to treat HIV-associated atherosclerosis.

\section{Discussion}

The goal of this study was to model Nef - calnexin cytoplasmic domain full interaction interface, and predict a range of possible small molecule inhibitors of this interaction. We have previously built models of Nef and calnexin cytoplasmic domain structures [13]. Quality Nef model is adequate but modelling of calnexin cytoplasmic domain was performed using less reliable templates. To enhance reliability of interaction prediction we have started by applying molecular dynamics simulation to create a new model, based on the previously built model of calnexin cytoplasmic domain structure.

We have then performed interaction prediction by Nef-calnexin docking utilising a new approach [32] and the QASDOM server developed by us and enabling enhanced interaction predictions from global docking data [26]. Using QASDOM server, we have obtained maps of predicted Nef-calnexin contacts, representative model structures of the complex, and location of the representative reciprocal interaction sites. Subsequently, we have used the 
predicted sites to carry out structure-based virtual screening and identified small-molecule drugs that bind to these sites and thus can potentially inhibit Nef-calnexin interaction.

Small molecule compounds predicted for calnexin sites A and $\mathbf{B}$ are located on the surface, but for the compounds docked to site $\mathbf{E}$ a major part of the molecules was positioned deep in the cleft in calnexin structure, with smaller part protruding above the molecular surface (Fig. 4B). It is possible that such positioning can result in the compounds binding to site $\mathbf{E}$ not only disrupting interaction with Nef, but also stabilising the structure of calnexin. In the full virtual screening results list, there are compounds with high affinity being predicted for more that one site; one compound was predicted to have high affinity for calnexin sites A-B and Nef site II, two compounds with predicted affinity for calnexin site B and Nef site II, and one compound with predicted affinity for Nef sites II and II-III (Tables S1 and S2). These compounds should be prioritised for experimental testing as potential drug candidates.

A rather remarkable result of virtual screening is that the compound $1[(7-\mathrm{oxo}-7 \mathrm{H}-$ benz[de]anthracene-3-yl)amino]anthraquinone (ZINC03953858, NSC13987), identified by us in previous virtual screening as a compound potentially blocking the Nef N-terminal binding site I (LYS7) interacting with calnexin, has been also identified in this study as potentially blocking the calnexin site B (Fig. S5). Since the NSC13987 compound was shown experimentally to rescue Nef-inhibited cholesterol efflux by inhibiting Nef-calnexin interaction [13], we can hypothesize that the compound disrupts this interaction by binding to both Nef and calnexin reciprocal interaction sites. To further optimize this effect, we synthesised a derivative of NSC13987, AMS-55. This compound potently inhibited the effect of Nef on ABCA1, reversing Nef-mediated downregulation of plasma membrane ABCA1 (the portion of the transporter responsible for cholesterol efflux) to levels observed in control untreated cells Fig. 5.

\section{Conclusions.}

The emphasis of this study was on modelling the full interaction interface in the complex of Nef with cytoplasmic domain of calnexin, aiming at subsequent identification of new compounds disrupting this interaction. We have modelled reciprocal binding sites in calnexin and Nef structures, and predicted small molecule compounds that can potentially inhibit this interaction by blocking the binding sites. Importantly, virtual screening confirmed the inhibitory potential of the previously identified drug NSC13987 [13], and identified a number of new compounds. Experimental testing of optimized analogue of NSC13987, AMS-55, demonstrated potent activity of this compound in reversing the effect of Nef on ABCA1. These findings provide a platform for searching for new therapeutic agents to treat HIV-associated co-morbidities, and we are planning to conduct extensive experimental testing of these compounds in our future work.

\section{Supplementary Material}

Refer to Web version on PubMed Central for supplementary material. 


\section{Acknowledgements}

AAAd conceived the project, coordinated the study and drafted the paper with contributions from AAAn, YT, MIB and AAM. AAAn, YT, YVK and AAAd performed computations and developed software. AK and TP conducted experiments. AAAd and MIB analysed the results with contributions from AAAn, YT, and TP. AAM and MIB coordinated the study. All authors read and approved the final manuscript. The study was funded by the Russian Foundation for Basic Research grant 17-54-30021 and NIH grant R01 NS102163.

\section{References}

1. d'Ettorre G, Ceccarelli G, Pavone P, Vittozzi P, De Girolamo G, Schietroma I, et al. What happens to cardiovascular system behind the undetectable level of HIV viremia? AIDS Res Ther 2016; 13:21. [PubMed: 27127532]

2. Mujawar Z, Rose H, Morrow MP, Pushkarsky T, Dubrovsky L, Mukhamedova N, et al. Human immunodeficiency virus impairs reverse cholesterol transport from macrophages. PLoS Biol 2006; 4:e365. [PubMed: 17076584]

3. Pushkarsky T, Shilov E, Kruglova N, Naumann R, Brichacek B, Jennelle L, et al. Short Communication: Accumulation of Neutral Lipids in Liver and Aorta of Nef-Transgenic Mice. AIDS Res Hum Retroviruses 2016; 33:57-60. [PubMed: 27649790]

4. Asztalos BF, Mujawar Z, Morrow MP, Grant A, Pushkarsky T, Wanke C, et al. Circulating Nef Induces Dyslipidemia in Simian Immunodeficiency Virus-Infected Macaques by Suppressing Cholesterol Efflux. J Infect Dis 2010; 202:614-623. [PubMed: 20617930]

5. Dubrovsky L, Duyne RV, Senina S, Guendel I, Pushkarsky T, Sviridov D, et al. Liver X receptor agonist inhibits HIV-1 replication and prevents HIV-induced reduction of plasma HDL in humanized mouse model of HIV infection. Biochem Biophys Res Commun 2012; 419:95-98. [PubMed: 22326260]

6. Cui HL, Ditiatkovski M, Kesani R, Bobryshev YV, Liu Y, Geyer M, et al. HIV protein Nef causes dyslipidemia and formation of foam cells in mouse models of atherosclerosis. FASEB J 2014; 28:2828-2839. [PubMed: 24642731]

7. Raymond A d., Campbell-Sims T c., Khan M, Lang M, Huang M b., Bond V c., et al. HIV Type 1 Nef Is Released from Infected Cells in CD45+ Microvesicles and Is Present in the Plasma of HIVInfected Individuals. AIDS Res Hum Retroviruses 2010; 27:167-178. [PubMed: 20964480]

8. Khan MB, Lang MJ, Huang M- B, Raymond A, Bond VC, Shiramizu B, et al. Nef exosomes isolated from the plasma of individuals with HIV-associated dementia (HAD) can induce A $\beta 1-42$ secretion in SH-SY5Y neural cells. J Neurovirol 2016; 22:179-190. [PubMed: 26407718]

9. Gupta MK, Kaminski R, Mullen B, Gordon J, Burdo TH, Cheung JY, et al. HIV-1 Nef-induced cardiotoxicity through dysregulation of autophagy. Sci Rep 2017; 7:8572. [PubMed: 28819214]

10. Jennelle L, Hunegnaw R, Dubrovsky L, Pushkarsky T, Fitzgerald ML, Sviridov D, et al. HIV-1 Protein Nef Inhibits Activity of ATP-binding Cassette Transporter A1 by Targeting Endoplasmic Reticulum Chaperone Calnexin. J Biol Chem 2014; 289:28870-28884. [PubMed: 25170080]

11. Chevet E, Wong HN, Gerber D, Cochet C, Fazel A, Cameron PH, et al. Phosphorylation by CK2 and MAPK enhances calnexin association with ribosomes. EMBO J 1999; 18:3655-3666. [PubMed: 10393181]

12. Schrag JD, Bergeron JJM, Li Y, Borisova S, Hahn M, Thomas DY, et al. The Structure of Calnexin, an ER Chaperone Involved in Quality Control of Protein Folding. Mol Cell 2001; 8:633-644. [PubMed: 11583625]

13. Hunegnaw R, Vassylyeva M, Dubrovsky L, Pushkarsky T, Sviridov D, Anashkina AA, et al. Interaction Between HIV-1 Nef and Calnexin: From Modeling to Small Molecule Inhibitors Reversing HIV-Induced Lipid Accumulation. Arterioscler Thromb Vasc Biol 2016; 36:1758-1771. [PubMed: 27470515]

14. Phillips JC, Braun R, Wang W, Gumbart J, Tajkhorshid E, Villa E, et al. Scalable Molecular Dynamics with NAMD. J Comput Chem 2005; 26:1781. [PubMed: 16222654]

15. Humphrey W, Dalke A, Schulten K. VMD: Visual molecular dynamics. J Mol Graph 1996; 14:3338. [PubMed: 8744570] 
16. MacKerell AD, Bashford D, Bellott M, Dunbrack RL, Evanseck JD, Field MJ, et al. All-atom empirical potential for molecular modeling and dynamics studies of proteins. J Phys Chem B 1998; 102:3586-3616. [PubMed: 24889800]

17. Mackerell AD, Feig M, Brooks CL. Extending the treatment of backbone energetics in protein force fields: Limitations of gas-phase quantum mechanics in reproducing protein conformational distributions in molecular dynamics simulations. J Comput Chem 2004; 25:1400-1415. [PubMed: 15185334]

18. Price DJ, Brooks CL. A modified TIP3P water potential for simulation with Ewald summation. J Chem Phys 2004; 121:10096-10103. [PubMed: 15549884]

19. Jorgensen WL, Chandrasekhar J, Madura JD, Impey RW, Klein ML. Comparison of simple potential functions for simulating liquid water. J Chem Phys 1983; 79:926-935.

20. Martyna GJ, Tobias DJ, Klein ML. Constant pressure molecular dynamics algorithms. J Chem Phys 1994; 101:4177-4189.

21. Feller SE, Zhang Y, Pastor RW, Brooks BR. Constant pressure molecular dynamics simulation: The Langevin piston method. J Chem Phys 1995; 103:4613-4621.

22. Hunter JD. Matplotlib: A 2D Graphics Environment. Comput Sci Eng 2007; 9:90-95.

23. Oliphant TE. Python for Scientific Computing. Comput Sci Eng 2007; 9:10-20.

24. R Core Team. R: a language and environment for statistical computing. Foundation for Statistical Computing, Vienna, Austria 2014http://www.R-project.org/

25. Grant BJ, Rodrigues APC, ElSawy KM, McCammon JA, Caves LSD. Bio3d: an R package for the comparative analysis of protein structures. Bioinformatics 2006; 22:2695-2696. [PubMed: 16940322]

26. Anashkina AA, Kravatsky Y, Kuznetsov E, Makarov AA, Adzhubei AA. Meta-server for automatic analysis, scoring and ranking of docking models. Bioinformatics 2018; 34:297-299.

27. Trott O, Olson AJ. AutoDock Vina: improving the speed and accuracy of docking with a new scoring function, efficient optimization and multithreading. J Comput Chem 2010; 31:455-461. [PubMed: 19499576]

28. Irwin JJ, Sterling T, Mysinger MM, Bolstad ES, Coleman RG. ZINC: A Free Tool to Discover Chemistry for Biology. J Chem Inf Model 2012; 52:1757-1768. [PubMed: 22587354]

29. Mukhamedova N, Fu Y, Bukrinsky M, Remaley AT, Sviridov D. The role of different regions of ATP-binding cassette transporter A1 in cholesterol efflux. Biochemistry (Mosc) 2007; 46:93889398.

30. Adzhubei AA, Sternberg MJ, Makarov AA. Polyproline-II Helix in Proteins: Structure and Function. J Mol Biol 2013; 425:2100-2132. [PubMed: 23507311]

31. Esipova NG, Tumanyan VG. Omnipresence of the polyproline II helix in fibrous and globular proteins. Curr Opin Struct Biol 2017; 42:41-49. [PubMed: 27815983]

32. Adzhubei AA, Anashkina AA, Makarov AA. Left-handed polyproline-II helix revisited: proteins causing proteopathies. J Biomol Struct Dyn 2017; 35:2701-2713. [PubMed: 27562438] 

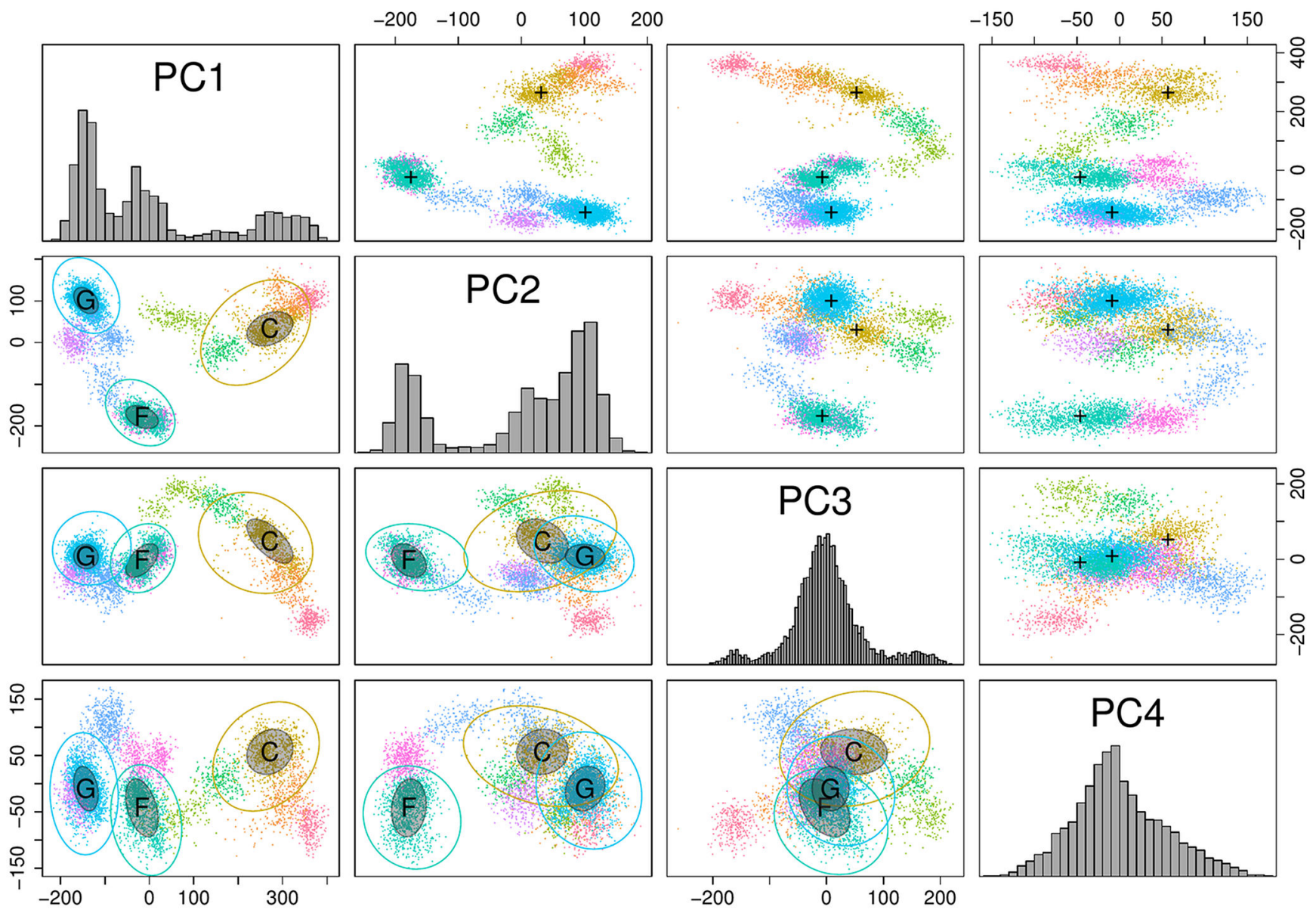

Figure 1.

Pairs plot matrix of calnexin cytoplasmic domain conformations classification in the space of four first principal components PC1-PC4. Diagonal contains histograms of corresponding PCs. Each $(i, j)^{\text {th }}$ panel off diagonal shows a projection of four-dimensional PC space, where each point represents a conformation, onto $(\mathrm{PC} i, \mathrm{PC} j$ ) plane. Points belonging to different groups are colored differently. Lower-left half also contains names, variances (shown as small shaded 1-sigma ellipses), and elliptical hulls of three groups with the largest population. Cross marks on upper-right half of the matrix denotes positions of representative conformations chosen from these groups. 

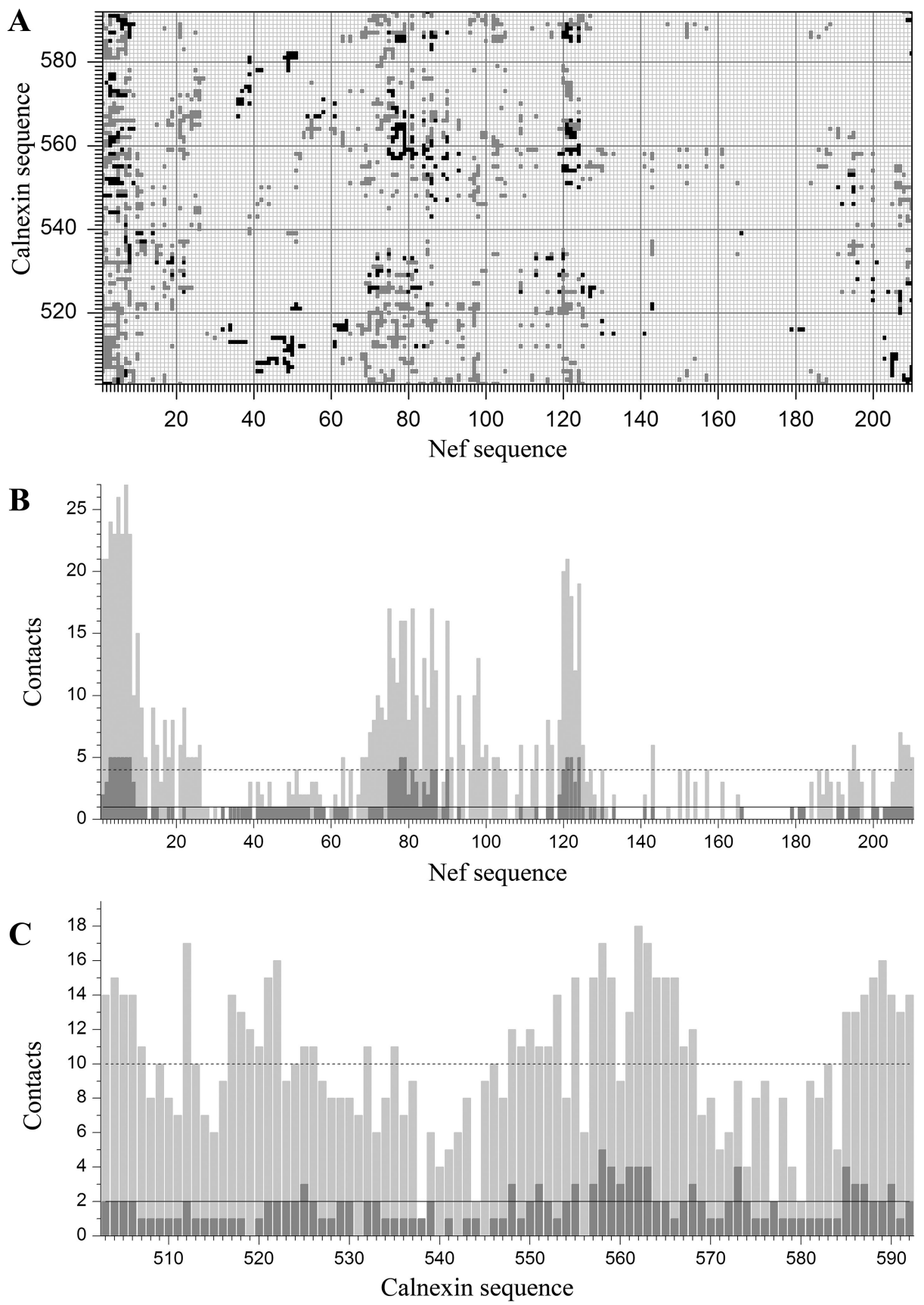

Figure 2.

(A) Map of Nef - calnexin atomic interactions calculated with QASDOM server. Contacts in the full dataset of Nef - calnexin docking models are shown by light gray. Darker colour represents the subset of 'good' docking models, selected according to the criteria set out in Materials and Methods section. (B and C) Linear clusters of residue interactions for Nef and calnexin sequences. Broken and solid lines show medians for contacts in the full (light gray) and 'good' (dark gray) datasets accordingly. 


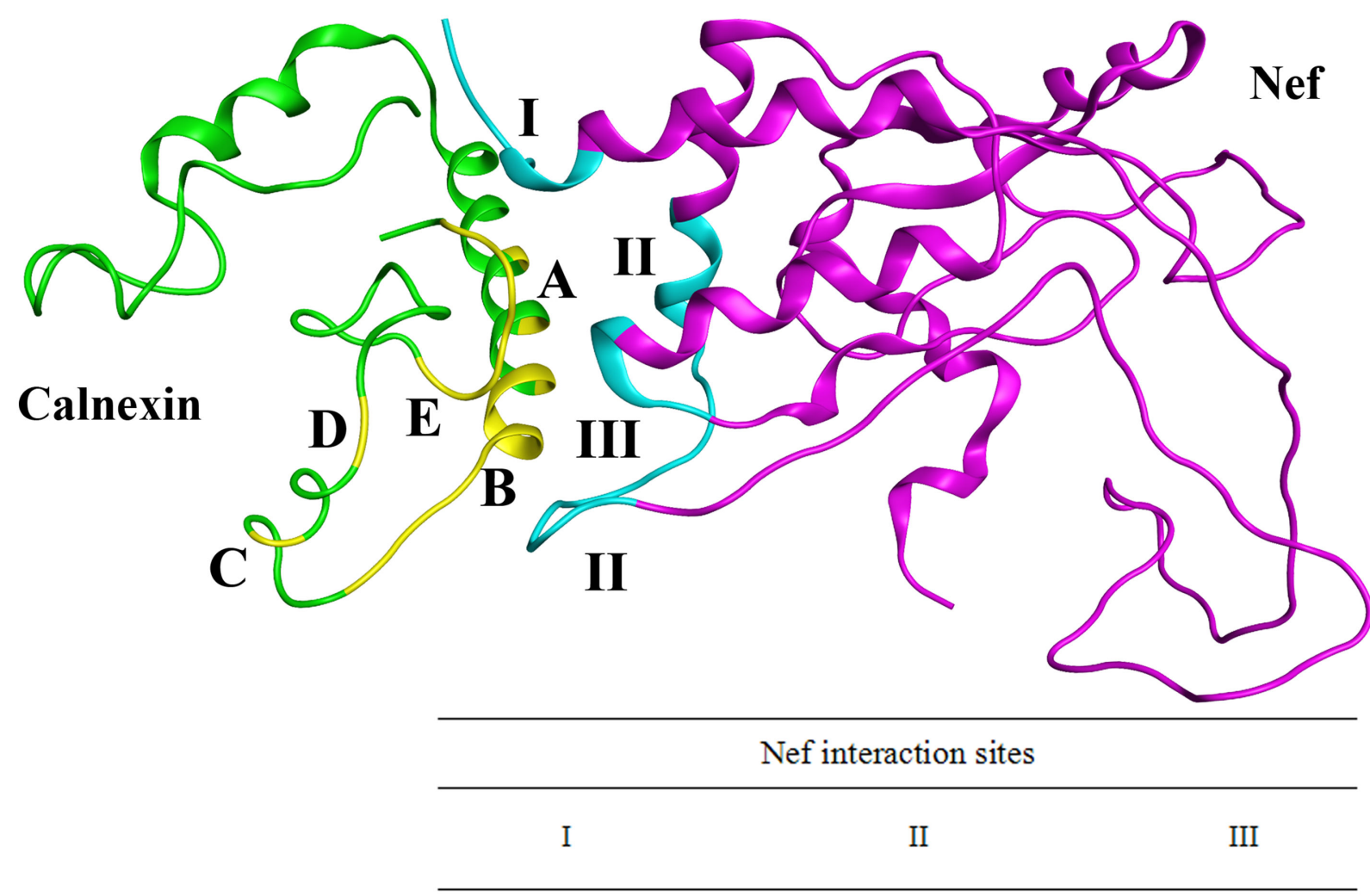

1-MGGKWSKRS-9 75-RPQVPLRPMTYKAALD-90 121-TQGY-124

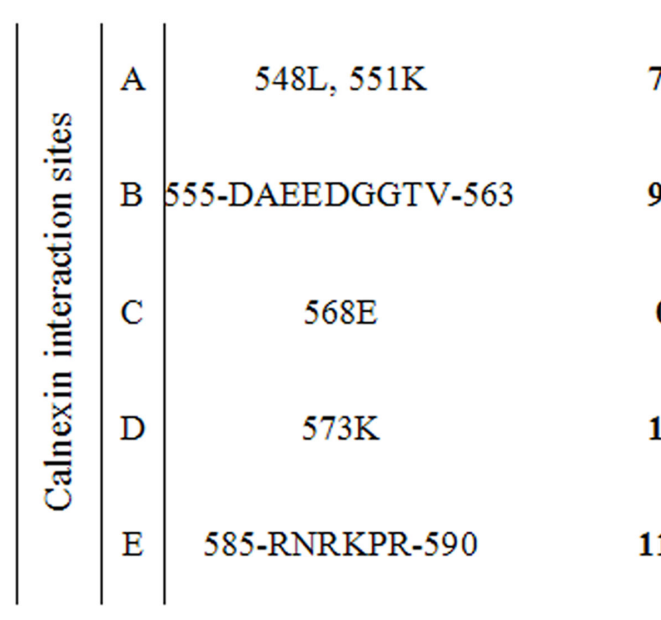

75

90

0

13

113
7

314

14

12

60
10

131

0

0

47

Figure 3.

Nef-calnexin interaction model created using QASDOM server from the data of global docking. Calnexin is shown in green, Nef in magenta. Reciprocal interaction sites in calnexin and Nef are shown in yellow and cyan. The panel shows atomic interactions between representative reciprocal sites in calnexin and Nef. Data from the Nef - calnexin map of contacts. 

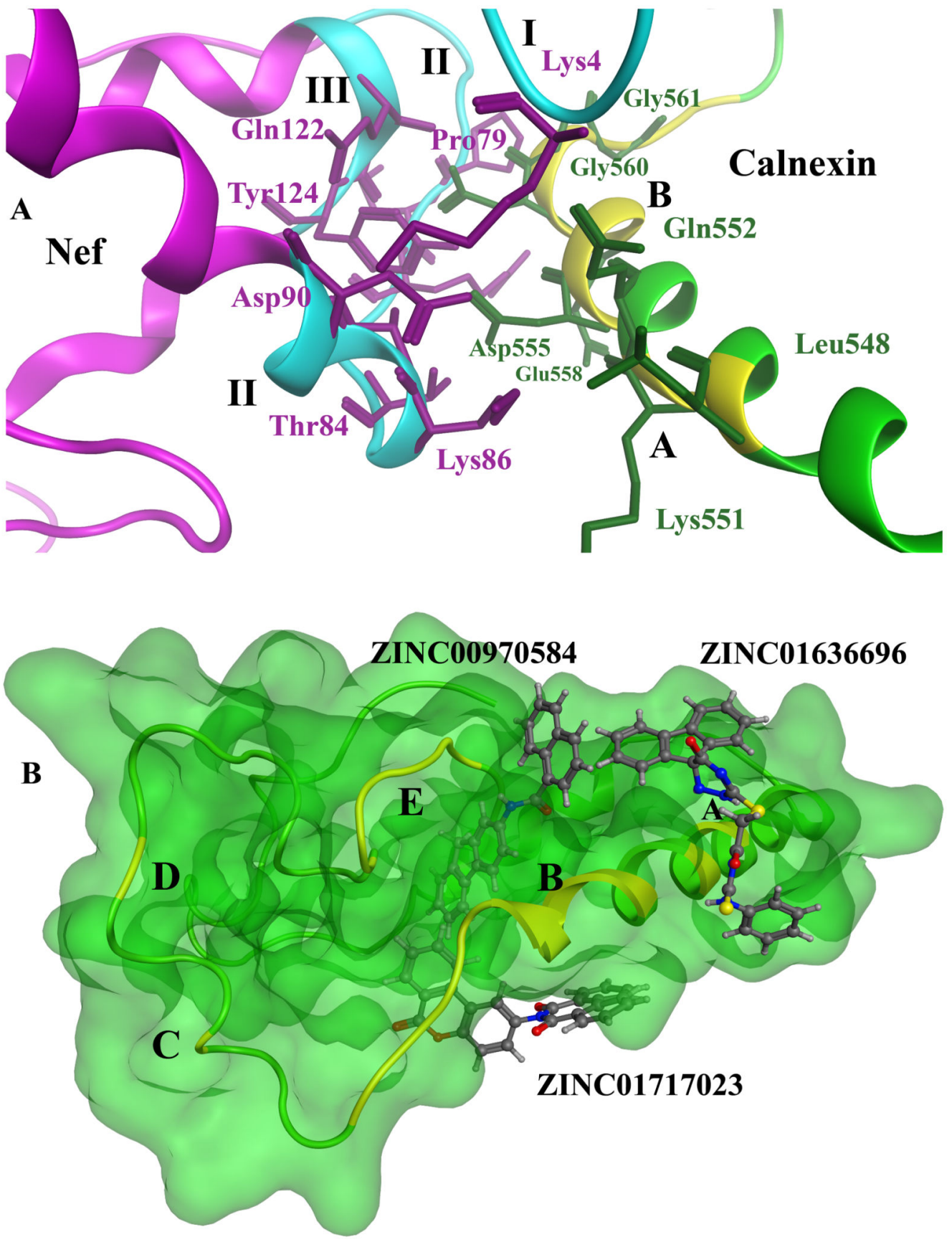

Figure 4.

Calnexin structure from Nef-calnexin complex model selected using QASDOM ranking from a range of models built by global docking. (A) Close-up of the Nef-calnexin complex model showing residues of the binding sites $\mathbf{A}$ and $\mathbf{B}$ in calnexin interacting with Nef sites $\mathbf{I}$, II and III. The sites which were covered by virtual screening in this or previous studies are marked in yellow for calnexin, and in cyan for Nef. (B) Calnexin structure with three smallmolecule compounds ZINC01636696, ZINC01717023 and ZINC00970584 predicted by 
virtual screening to bind to sites $\mathbf{A}, \mathbf{B}$ and $\mathbf{E}$. The compounds can potentially disrupt interaction of the two proteins. 
A

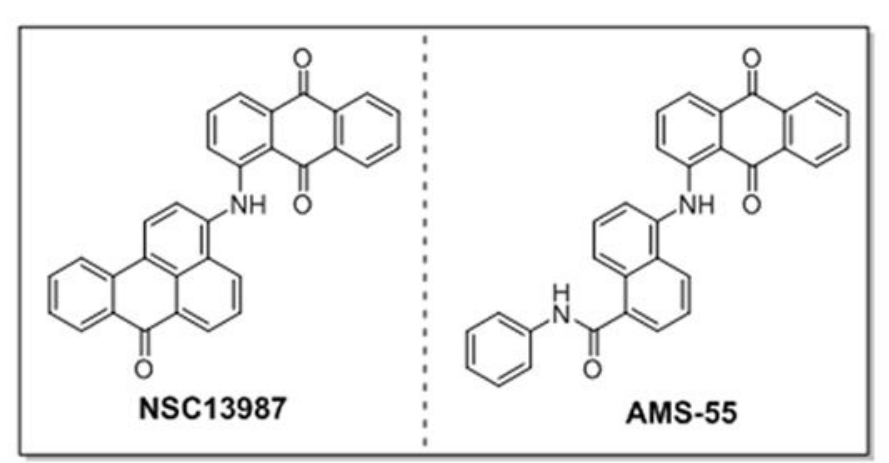

B

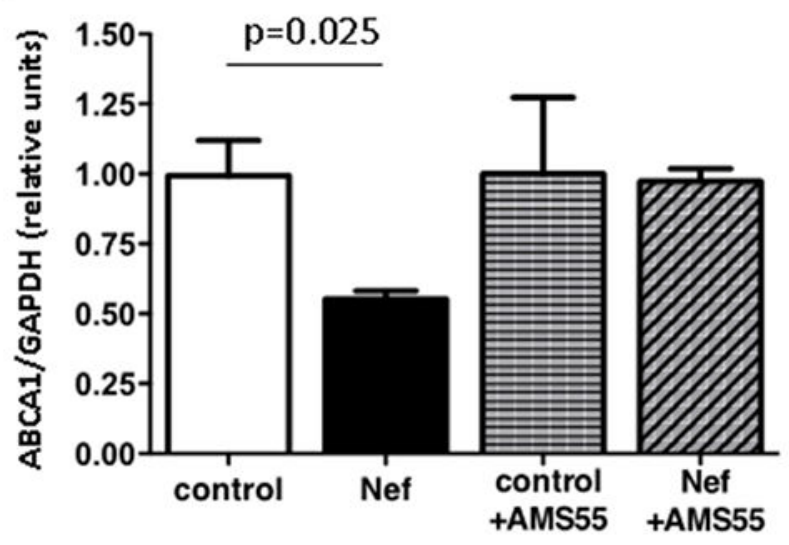

Figure 5.

(A) Structural models of the parent compound, NSC13987, and its analogue, AMS-55. (B) Experimental testing of AMS-55. HeLa-ABCA1 were transfected with Nef-expressing or empty vector (control) and treated or not with AMS-55 $(1 \mu \mathrm{M})$. ABCA1 and GAPDH were assayed by Western blot, and results are presented as ABCA1 to GAPDH ratio in 3 independent experiments. P value was calculated by Student's unpaired $t$ test. 